\title{
Radiographic and prenatal ultrasound features of perinatal lethal hypophosphatasia - differentia- tion from osteogenesis imperfecta type II
}

\author{
S Wiebe, MD, FRCPC \\ Department of Medical Imaging, Royal University Hospital and University of \\ Saskatchewan, Saskatoon, Canada \\ I Suchet, MB BCh, FRCPC \\ Regina Qu'Appelle Health Region, Regina, Saskatchewan, Canada \\ E G Lemire, MD, PhD, FRCPC, FCCMG, FACMG \\ Division of Medical Genetics, Department of Paediatrics, Royal University \\ Hospital and University of Saskatchewan, Saskatoon, Canada
}

\section{Introduction}

Hypophosphatasia is a genetic disorder that is characterised by deficient activity of the enzyme alkaline phosphatase. Clinically, it can present as a lethal infantile form, an intermediate childhood form with rachitic changes and premature deciduous tooth loss or a mild adult form with a tendency for fractures and bone pain. Both autosomal-dominant and recessive inheritance has been reported for the mild forms, but only autosomal-recessive inheritance has been implicated in the lethal infantile form. ${ }^{1}$ Our case presented as a severe skeletal dysplasia antenatally and was carried to term. The baby succumbed shortly after delivery. Initially, the radiographic findings were thought to be consistent with the severe lethal form of osteogenesis imperfecta (OI) type IIA. Investigations performed at birth including genetic testing, conclusively determined that the correct diagnosis was the perinatal lethal form of hypophosphatasia.

Differentiating these two entities can be difficult given the variability of findings and the wide spectrum of severity (Tables I and II). Cases of perinatal lethal hypophosphatasia commonly have markedly reduced ossification and may have complete absence of ossification of some bones. The type of ossification defect in OI type IIA is slightly different in that there is usually severe diffuse demineralisation of the skull $^{2}$ (Fig. 1) instead of the demineralisation with 'island-like' centres of ossification in the frontal, parietal and occipital bones which is seen in hypophosphatasia $^{3}$ (Fig. 2). Also, in OI type IIA there is overall diffuse osteopenia (demineralised bones) and usually not the near complete lack of mineralisation in individual bones with adjacent more densely mineralised bones. The latter is a feature of hypophosphatasia (Figs 3 and 4). One specific prenatal sonographic area to help differentiate between the two is the infant's hands. The bones of the hands are sonolucent in patients with hypophosphatasia but are echogenic in patients with osteogenesis imperfecta. ${ }^{4}$

\section{Case report}

This was a planned pregnancy for a healthy 33-year-old mother and 38year-old father. There were 2 prior uncomplicated pregnancies that gave rise to a healthy son and daughter. The mother was on paroxetine hydrochloride, fluticasone propionate and doxylamine succinate-pyridoxine hydrochloride during the pregnancy. There were no known exposures to any teratogens. Folic acid supplementation was initiated pre-conceptionally. The family history was unremarkable. Parents were both Caucasians and of European descent. They were not known to be consanguineous.

At 17.8 weeks' gestation, a routine antenatal ultrasound identified multiple skeletal abnormalities. There was complete absence of cranial ossification and severe shortening of all four limbs, especially proximally. All the long bones were found to be less than 2 standard deviations below the mean for gestational age. The long bones were thickened and bent, compatible with callous formation. Vertebral ossification appeared normal. The remainder of the fetal anatomy was unremarkable and there was adequate amniotic fluid. The working diagnosis was OI type II, however hypophosphatasia and achondrogenesis were also considered in the differential diagnosis. Maternal serum screening was declined and amniocentesis was not offered. The couple declined to terminate the pregnancy.

Repeat ultrasounds in the 27th and 36th week of gestation identified similar skeletal findings. Other findings included ventricular dilatation $(22 \mathrm{~mm})$ at 27 weeks and polyhydramnios, platyspondyly and a small thoracic cavity at 36 weeks' gestation.

\begin{tabular}{|lll|}
\hline \multicolumn{4}{|l}{ Table I. Radiographic features of osteogenesis imperfecta type IIA and the perinatal form of hypophosphatasia } \\
\hline Skull & Osteogenesis imperfecta type IIA & \multicolumn{1}{l}{ Hypophosphatasia, perinatal form } \\
Ribs & Caput membranaceum, wormian bones & $\begin{array}{l}\text { Caput membranaceum, absence of ossification of calvaria and bones of the } \\
\text { skull base }\end{array}$ \\
Vertebrae & Short, thick ribs with continous beading & Short, thin ribs \\
Long bones & Flat and irregularly deformed & Poorly ossified, especially neural arches \\
& Thick, short, crumpled shafts, multiple & Tubular bones, absent ossification of whole bones, diaphyseal \\
Other & fractures, callous formation & spurs, punched out metaphyseal irregularities, kyphomelia \\
& Generalised hypomineralisation & Generalised hypomineralisation
\end{tabular}




\begin{tabular}{|ll|}
\hline \multicolumn{2}{|c|}{ Table II. Sonographic findings of osteogenesis imperfecta type IIA and the perinatal form of hypophosphatasia } \\
\hline Osteogenesis imperfecta type IIA & Hypophosphatasia, perinatal form \\
\hline Short limbs with wrinkled appearance of long bones & Short, bowed limbs \\
Multiple fractures with callus formation & Polyhydramnios \\
Demineralised calvarium & Small for gestational age \\
Abnormal skull shape & Decreased echogenicity of the bones \\
Small chest circumference with broad and irregular ribs & Prominent falx cerebri \\
Increased nuchal translucency & Underossification of vertebrae, neural arches and hands \\
\hline
\end{tabular}

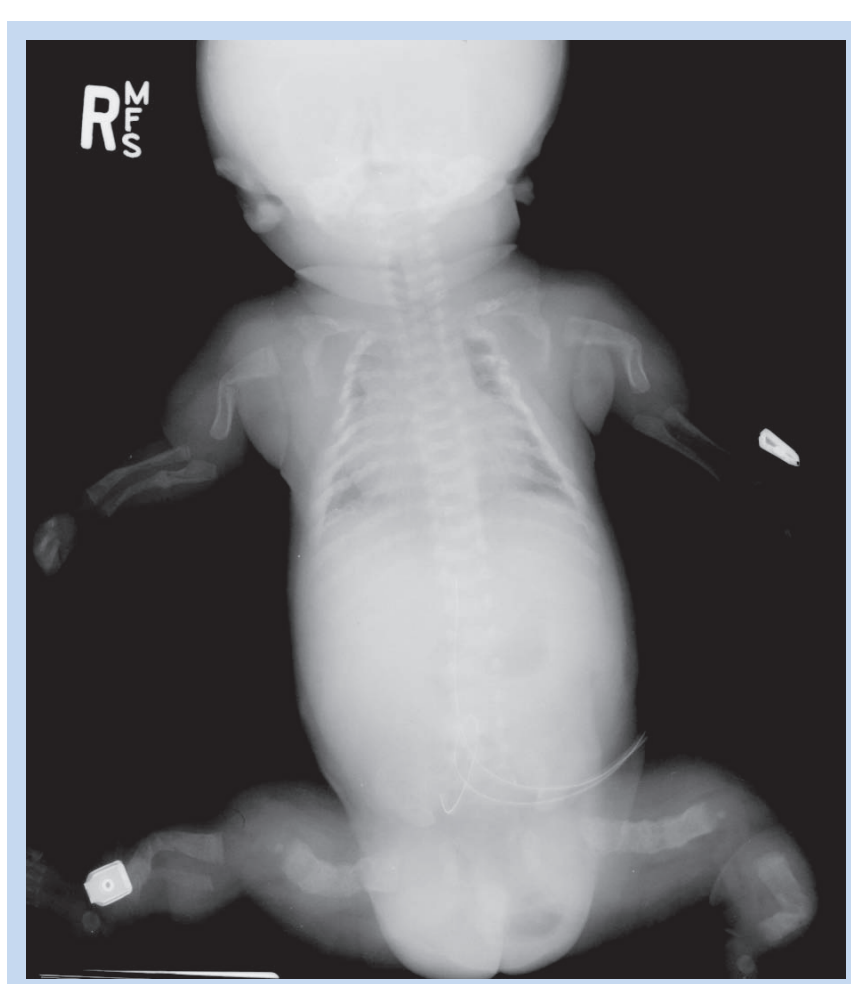

Fig. 1. AP view of a different child with the lethal form of osteogenesis imperfecta. Note the diffuse osteopenia and multiple fractures.

A male infant was delivered at term by vaginal delivery weighing $3.4 \mathrm{~kg}$. Compassionate care was provided and the infant succumbed within several minutes. Other growth parameters included a crown to heel length of $47 \mathrm{~cm}$ and a head circumference of $34 \mathrm{~cm}$. All limbs were visibly short, thickened, curved and measured much less than two standard deviations below the mean for gestational age. The chest appeared narrowed and the abdomen was protuberant. There were no other visible anomalies. The following investigations were requested: DNA banking for future studies, karyotype analysis, skin biopsy for collagen studies, skeletal X-ray survey, clinical photographs and a full autopsy examination.

Permission was not given for an internal examination on the postmortem. X-rays revealed abnormalities thought to be consistent with OI type II.A Chromosome studies revealed a normal male karyotype, 46 ,XY. Collagen studies from the fibroblasts did not reveal any abnor-

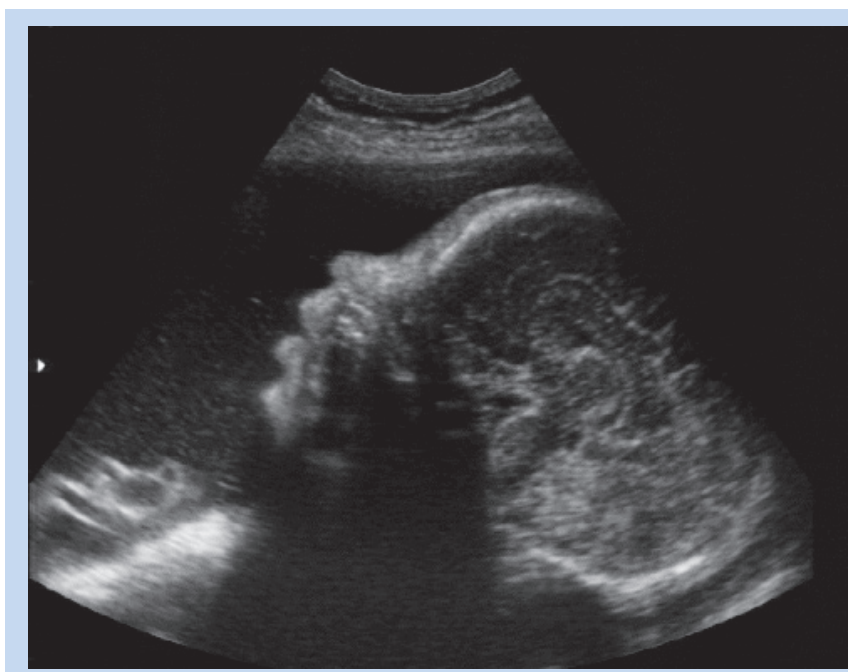

Fig. 2. Prenatal ultrasound of the head with lack of shadowing from the unossified skull shows better than normal visualisation of the brain due to unmineralised cranium.

mality in type I and type III pro-collagen and collagen molecules, thus excluding most cases of OI type II. A review of the X-rays suggested that the radiographic findings were more in keeping with hypophosphatasia. Mutation analysis was performed and two mutations (G863A and G1001A) in the alkaline phosphatase gene (ALPL) were identified. This is consistent with autosomal-recessive inheritance and the perinatal (lethal) form of hypophosphatasia. Both mutations resulted in glycine to aspartic acid substitutions in the mature protein. The parents were each subsequently confirmed to be heterozygous mutation carriers for hypophosphatasia.

\section{Discussion}

The perinatal lethal form of hypophosphatasia is an autosomal-recessive inborn error of bone metabolism. There is a deficiency of alkaline phosphatase activity in the bone which results in abnormal unossified bones with variable involvement. The prenatal ultrasound findings include short limbs with multiple deformities and a soft non-ossified calvarium, which allows the brain to be seen unobscured by the normally echogenic cranium. Polyhydramnios may be present. Postnatal radiographs will demonstrate a variable appearance of non-ossified bones, osteopenia and some segments of more normal ossification. The clavicles are usually spared in the infantile form. ${ }^{3}$ The cranial ossification is deficient 


\section{CASE REPORT}

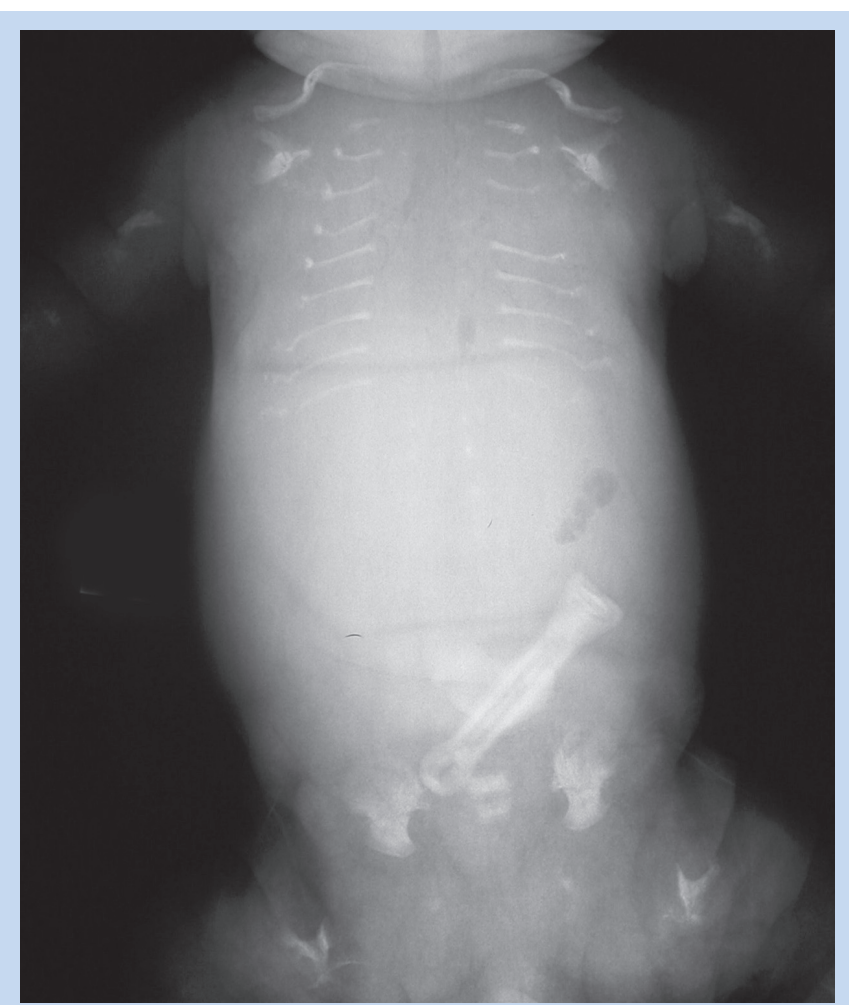

Fig. 3. Postnatal AP view of the chest and abdomen revealing lack of mineralisation of many vertebrae and abnormally thin irregular (fractured) ribs. More normal ossification of some of the sacral vertebrae.

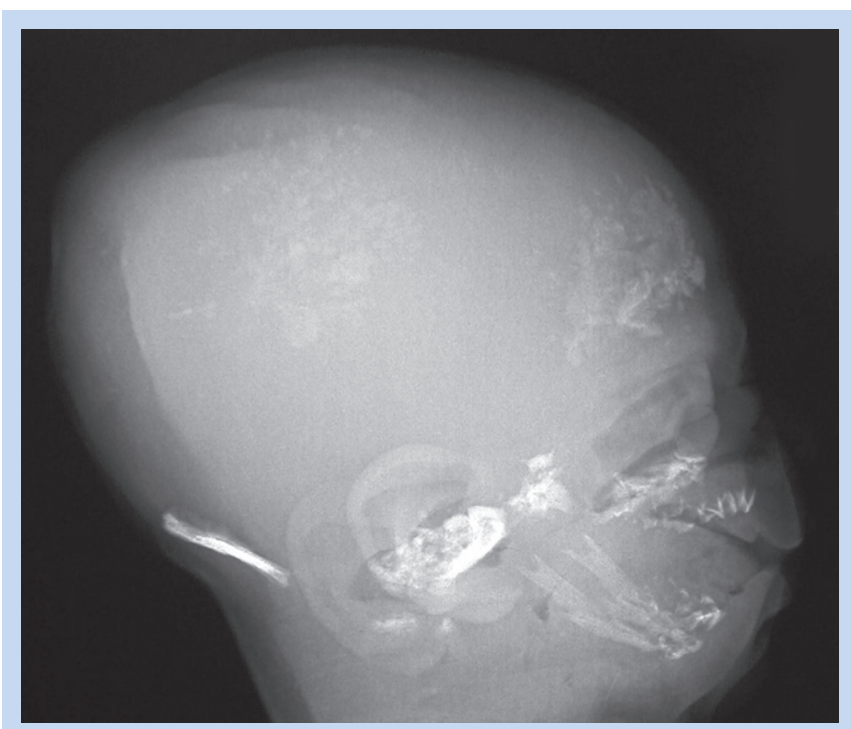

Fig. 4. Postnatal lateral view of the skull demonstrating diffuse lack of ossification and patchy island-like areas of ossification of the occipital bone, skull base, and to a lesser extent in the frontal and parietal bones.

but there may be 'island-like' areas of ossification as seen in our case. In some cases there are osteochondral projections at right angles to the mid-diaphysis of the long bones of the arms and legs which are called Bowder spurs ${ }^{1,3,5}$ and may be more specific for hypophosphatasia. Our case did not have Bowder spurs present however we did find sonolucent and radiolucent bones of the hands in keeping with the diagnosis of hypophosphatasia (Figs 5a and b, and Fig. 6).

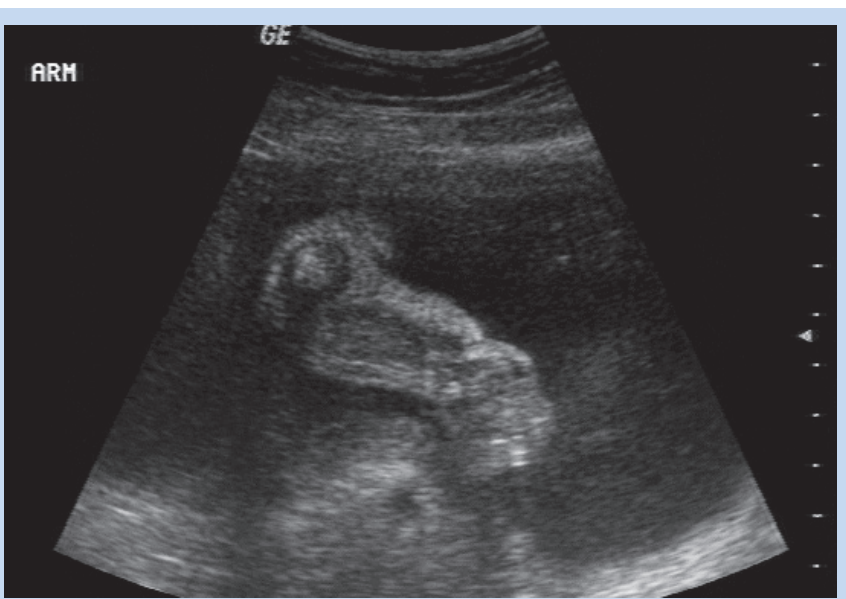

Figs $5 a$ and $b$. Prenatal ultrasound of the arm demonstrating short angulated bones in keeping with multiple fractures.

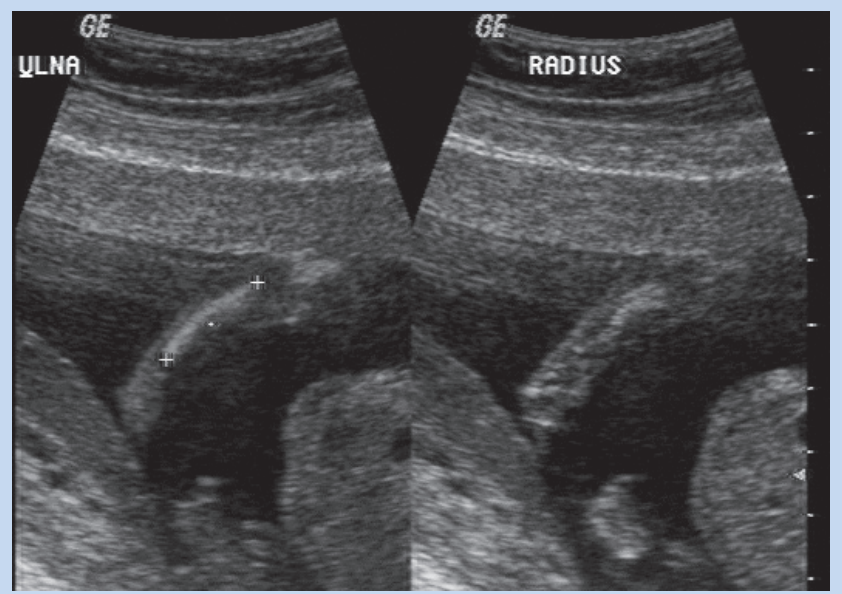

Fig. 5b.

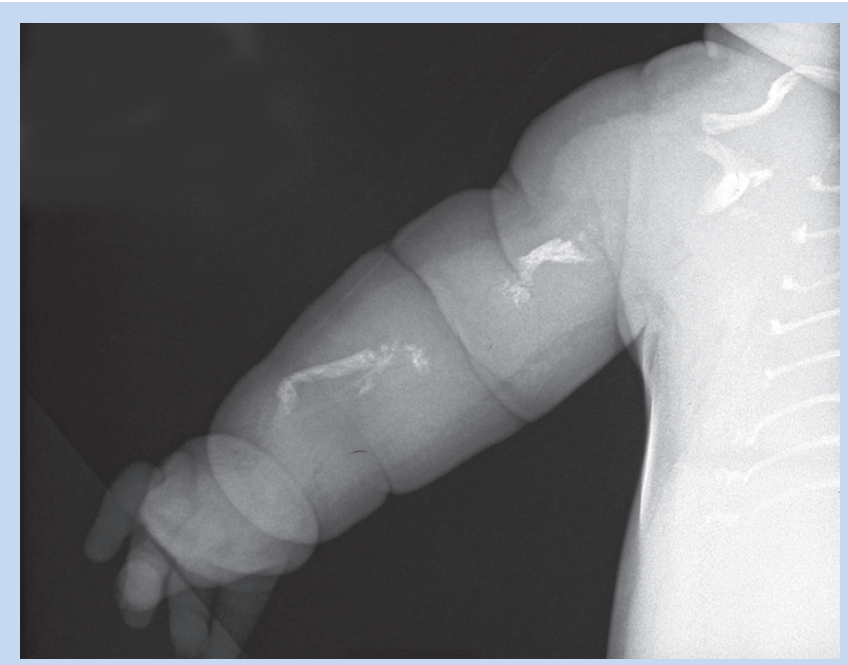

Fig. 6. Postnatal view of the arm with abnormal ossification of the short bones with multiple fractures present.

Differentiating these cases from OI type II can be difficult based on imaging because of the variability of findings and the overlap of the lack of ossification, multiple fractures, etc. Some of the radiographic features seen in our case direct us to favour hypohosphatasia, which was 


\section{CASE REPORT}

subsequently confirmed molecularly. This case clearly demonstrates the importance of performing cytogenetic, radiographic, pathologic and molecular investigations, whenever possible, for confirmation of the diagnosis when a skeletal dysplasia is suspected on antenatal ultrasound. Looking at the infant's hands on the prenatal ultrasound may help make the diagnosis of hypophosphatasia if the bones are sonolucent.

This couple had inappropriately received a low recurrence risk based on the diagnosis of OI type IIA. The perinatal lethal form of hypophosphatasia is inherited in an autosomal-dominant fashion and is associated with a $25 \%$ recurrence risk. Identification of the two alkaline phosphatase mutations in the infant and confirmation of carrier status in the parents now allows us to offer prenatal diagnostic testing for future pregnancies.

1. Taybi H, Lachman RS. Radiology of Syndromes, Metabolic Disorders, and Skeletal Dysplasias. 4th ed. Philadelphia: Mosby, 1996: 628-630

2. Taybi H, Lachman RS. Radiology of Syndromes, Metabolic Disorders, and Skeletal Dysplasias. 4th ed. Philadelphia: Mosby, 1996: 876-881.

3. Kuhn JP, Slovis TL, Haller JO. Caffey's Pediatric Diagnostic Imaging. 10th ed. St. Louis: Mosby/Elsevier Science, 2004: 2165-2167, 2264-2265.

4. Tongsons T, Pongsatha S. Early prenatal sonographic diagnosis of congenital hypophosphotasia. Ultrasound Obstet Gyenecol 2000; 15:252-255.

5. Shohat M, Rimoin DL, Gruber HE, Lachman RS. Perinatal lethal hypophosphatasia: clincal, radiographic and morphological findings. Pediar Radiol 1991; 21:421-427.

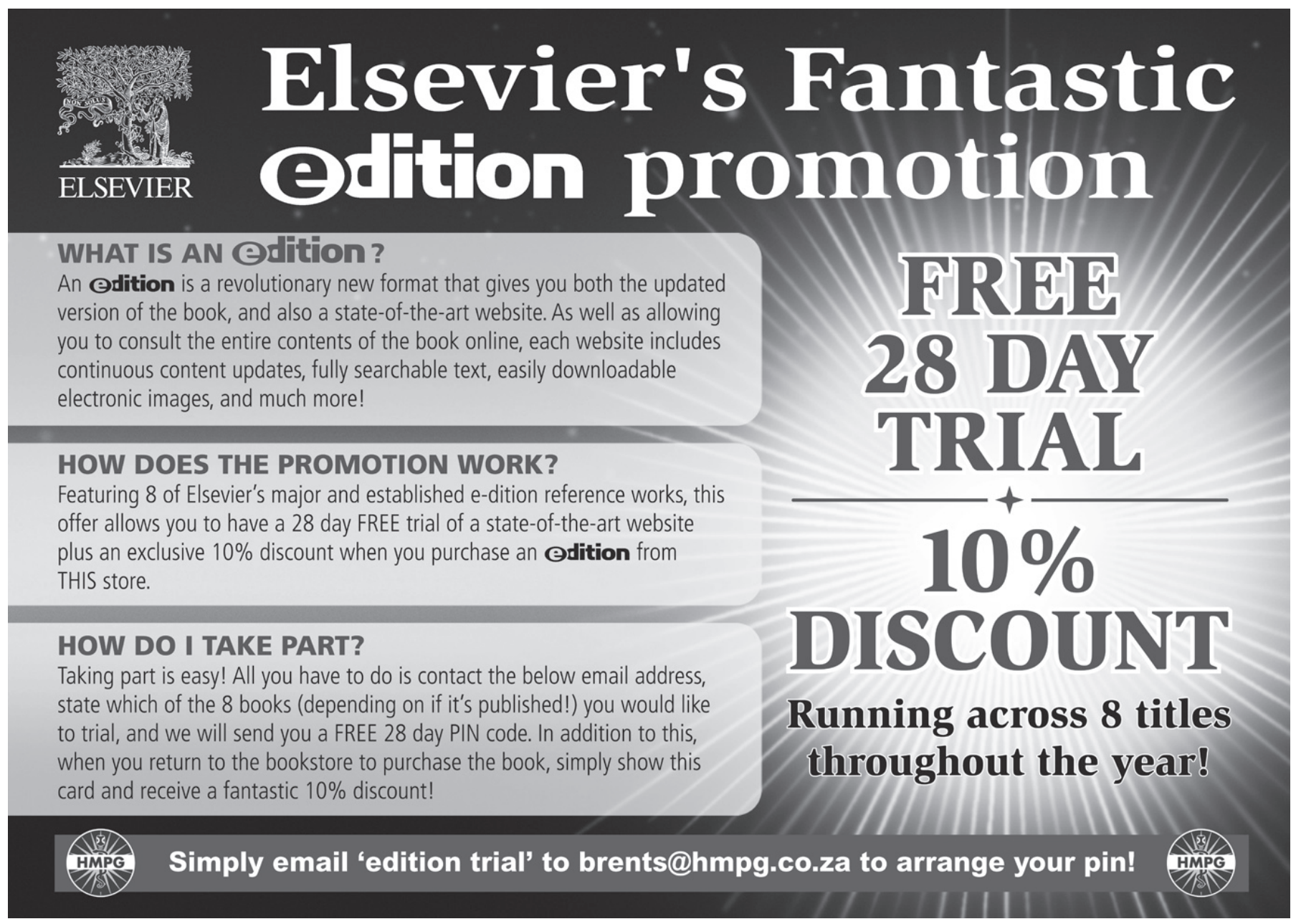

